\title{
EVALUACION RADIOISOTOPICA EN CARDIOLOGIA
}

\section{Drs. Teresa Massardo V(1, 2), Patricio González $E^{(1,2), ~ J o s e ́ ~ C a n e s s a ~ G(2) . ~}$}

1. Medicina Nuclear. Departamento de Medicina del Hospital Clínico Universidad de Chile.

2. Medicina Nuclear, Centro Radiologico Fleming.

\begin{abstract}
The current applications of nuclear cardiology techniques are reviewed. Coronary artery disease is the most important and prevalent cardiovascular problem in most developed countries and also in Chile. Different approaches can be employed for its diagnosis and prognosis, as well as for risk stratification and preoperative evaluation. Myocardial infarction assessment and ischemia recognition with radionuclide perfusion images are also reviewed, including new protocols applying functional parameters addition. Viability detection after myocardial infarction or in patients with ischemic cardiomyopathy are discussed. Stress protocols with the use of exercise or pharmacological action for ischemia production, the diagnostic value of perfusion SPECT and the use of Thallium-201, TC-99mSestamibi and metabolic images with Fluorine18Fluordeoxyglucose are also mentioned.
\end{abstract}

Key words: Coronary artery disease, myocardial infarction, nuclear cardiology.

Resumen: Se revisan las aplicaciones comunes de las técnicas de medicina nuclear en cardiología. La más importante y prevalente patología cardiovascular en países desarrollados y también en Chile es la enfermedad coronaria. Diferentes aproximaciones pueden ser empleadas para su estudio, pronóstico, estadificación de riesgo y evaluación preoperatoria. Se revisan, además, el infarto miocárdico y el reconocimiento de isquemia con estudio de perfusión incluyendo nuevos protocolos que aplican producción de isquemia; el valor diagnóstico del SPECT de perfusión, el uso de Talio; Tc-99m Sestamibi e imágenes metabólicas con Flúor-18-

\footnotetext{
Massardo T. y cols. Evaluación radioisotópica en cardiología. Rev Chil Radiol 2002; 8:48-52.

Correspondencia: Dra. Teresa Massardo V.

Sección Medicina Nuclear, Departamento de Medicina Hospital Clínico Universidad de Chile, Santos Dumont 999-1E. Fono :562-7770569

Email: tmassardo@ns.hospital.uchile.cl
}

Fluorodeoxiglucosa son también mencionadas.

Palabras claves: Enfermedad coronaria, Viabilidad miocárdica, Perfusión miocárdica, Función ventricular, Isquemia miocárdica, Infarto de miocardio.

\section{Introducción}

La cardiopatía o enfermedad coronaria (EC), es en la actualidad, de alta prevalencia en muchos países y en el nuestro es similar a la de países desarrollados. Las enfermedades cardiovasculares son la primera causa de muerte en adultos en Chile y su forma clásica de presentación es angina provocada por esfuerzo o en casos más graves angina de reposo; sin embargo, especialmente en diabéticos, el cuadro inicial puede ser un evento cardíaco mayor que incluyen infarto del miocardio o muerte. La forma más solapada de presentación con insuficiencia cardiaca congestiva (ICC) y enfermedad crónica de múltiple vaso ha sido reconocida últimamente, siendo la miocardiopatía isquémica la causal de al menos $1 / 3$ de todas las ICC.

La EC puede ser abordada de diversas maneras, tanto en su diagnóstico como en la evaluación de su pronóstico, incluyendo el posterior a procedimientos de revascularización. El clásico electrocardiograma basal (ECG) y el de esfuerzo, la monitorización continua con Holter, la ecocardiografía, los radionúclidos, incluyendo los estudios de función ventricular y perfusión miocárdica, la tomografía de positrones (PET) e incluso la resonancia magnética (RM) gatillada tienen, en mayor o menor grado, un papel reconocido. Sin embargo, el patrón de referencia continúa siendo la coronariografía. En la actualidad, la perfusión coronaria estudiada con TI-201 o Tc-99mSestamibi tienen un gran lugar en la evaluación no invasiva de la EC.

Los estudios de función ventricular con isótopos fueron muy utilizados hace algunos años, y su uso actual es en la evaluación objetiva de la función sistólica, específicamente fracción de eyección (FE) del ventrículo izquierdo (VI), en especial, en pacientes oncológicos con riesgo de cardiotoxicidad por 
antraciclinas o en casos de miocardiopatía isquémica o de otro origen, que deban ser sometidos a terapia invasiva. Además, se puede evaluar alteración de motilidad parietal y de volúmenes ventriculares.

La coronariografía junto a la ventriculografía de contraste ofrecen parámetros anatómicos relacionados al grado de estenosis coronaria, motilidad regional en reposo y también de la revascularización efectuada, evaluando la permeabilidad de puentes venosos o arteriales o el estado de los procedimientos de dilatación mediante angioplastía con balón con o sin implante de stents. Es aceptado que estenosis menores de $50 \%$ no tienen significado clínico y que estenosis mayores a $70 \%$ son enfermedad coronaria grave. Estos métodos no evalúan el flujo a nivel de la microvasculatura en las diversas condiciones existentes en la vida diaria.

\section{Principales indicaciones de los estudios de perfusión miocárdica}

La principal indicación es el screening en poblaciones de riesgo clínico intermedio de EC que comprende hombres de edad mediana con antecedentes de dislipidemia, tabaquismo y prueba de esfuerzo dudosos o dolor precordial atípico y mujeres perimenopáusicas con ECG de esfuerzo y/ o dolor no característico. El objetivo es seleccionar en forma más adecuada los requerimientos de coronariografía. La sensibilidad y especificidad de los estudios de perfusión con estrés en el diagnóstico de EC están alrededor de $80-85 \%$ en diversos trabajos (Tablas I y II).

\begin{tabular}{|c|c|c|}
\hline $\begin{array}{l}\text { TABLA I } \\
\text { Rendimiento } \\
\text { isquemia }\end{array}$ & ECT perfus & on provocación de \\
\hline & Cualitativo & Cuantitativo \\
\hline Sensibilidad & $89 \%$ & $90 \%$ \\
\hline Especificidad & $76 \%$ & $70 \%$ \\
\hline $\begin{array}{l}\text { (ACC/AHA/A } \\
1999)\end{array}$ & SIM Guid & J Am Coll Cardiol \\
\hline
\end{tabular}

TABLA II

Diagnóstico de EC en pacientes sin sesgo de referencia.

\begin{tabular}{lllr} 
& Planar & \multicolumn{1}{l}{ SPECT } & $n$ \\
\hline Sensibilidad & $89 \%$ & $89 \%$ & 839 \\
Especificidad/N R* & $91 \%$ & $73 \% / 90 \%$ & 1.901
\end{tabular}

Probabilidad clínica de EC $<5 \%$ (revisión de literatura) ${ }^{*} \mathrm{NR}=$ Normalcy rate

El valor pronóstico de los estudios de perfusión tiene gran utilidad en la práctica clínica. Se acepta que un estudio de perfusión en estrés-reposo normal, tiene una incidencia menor al $1 \%$ al año, de eventos cardíacos mayores, según los trabajos de Brown y cols. La validez de esta afirmación es por dos años, puesto que la EC es progresiva. La otra gran indicación es la evaluación de la EC conocida y la estadificación de riesgo, tanto para determinar su extensión y severidad como para definir tratamiento médico o revascularización coronaria. En forma habitual, pacientes que deben ser sometidos a cirugía no cardíaca son también evaluados con estudios de perfusión miocárdica preoperatoria.

En el infarto de miocardio (IM) los isótopos sirven para confirmar su presencia y magnitud, evaluar viabilidad, isquemia residual, resultados de revascularización y también, con fin pronóstico. Postinfarto, coexisten zonas de necrosis, isquemia, hibernación, atontadas (especialmente, si hubo reperfusión) e incluso áreas con perfusión normal. La revascularización permite mejorar la función regional y global del ventrículo izquierdo y puede mejorar la sobrevida a largo plazo. Es por esto que los métodos con capacidad de pesquisar viabilidad miocárdica tienen un papel importante en la actualidad. Un método más reciente para reconocer la viabilidad miocárdica es el uso de un metabolito marcado similar a la glucosa, la deoxiglucosa-Fluor18 (FDG). Su acumulación en tejido hipoperfundido y con alteración de motilidad funcional permite pesquisar viabilidad y otorga al paciente la posibilidad de la revascularización.

También, se plantean los estudios de perfusión en centros de alta complejidad y mayores recursos para decidir el ingreso a unidades coronarias en casos difíciles, y para estadificación, en síndromes coronarios agudos con probabilidad clínica baja o intermedia de riesgo. Se utilizan imágenes sólo en reposo con Sestamibi. Se obtiene información de presencia, localización, tamaño y severidad del infarto, sin embargo, ello requiere equipos en las unidades de emergencia y personal permanente. El Sestamibi por sus propiedades de mínima redistribución, permite estudiar el miocardio aplazando las imágenes en algunas horas, hasta obtener estabilización del paciente.

Valor Pronóstico Post-Infarto Miocárdico y en ICC

La existencia de isquemia residual en el territorio del infarto es un factor de riesgo, tal como la isquemia en otros territorios coronarios. El tamaño del infarto y su viabilidad son factores pronósticos significativos y se considera el riesgo clínico para la selección de pruebas invasivas. Beller, plantea un algoritmo de costo/beneficio en la toma de decisiones de acuerdo con guidelines recientes de infarto de miocardio.

La miocardiopatía isquémica es una patología frecuente entre los pacientes portadores de ICC, en 
quienes no se han presentado síntomas isquémicos. La revascularización coronaria extensa en quienes tengan aun músculo viable es una alternativa terapéutica final más al alcance que el transplante cardíaco. Puede pesquisarse la viabilidad, como ya se mencionó, con pruebas de perfusión miocárdica utilizando equipos de gama cámara tomográficas SPECT, con estudios de metabolismo de glucosa con equipos de positrones dedicados PET o con equipos SPECT con características especiales.

\section{Equipos usados en cardiología nuclear}

Gamacámaras SPECT: los estudios de perfusión miocárdica se pueden efectuar con equipos planares, pero su rendimiento es algo menor. EI SPECT es preconizado de elección por su capacidad de desproyectar los distintos territorios. Pueden gatillarse los estudios y obtener parámetros de motilidad con cine, función sistólica (fracción de eyección ventricular) y volúmenes ventriculares. Estos equipos cuentan con softwares complejos para analizar las imágenes en forma cuantitativa y compararlos con bases de datos normales. Con colimadores especiales o con características especiales de diseño, también pueden detectar los rayosgamma producto de la emisión de positrones en forma aislada o en coincidencia.

Equipos PET dedicados: Sólo sirven para estudios con isótopos emisores de positrones. Son de gran costo y complejos. Utilizan métodos de detección también basados en cristales emisores de luz al incidir en ellos un fotón en coincidencia, al igual que los anteriores.

\section{Características principales de los radioisótopos usados en cardiología nuclear: Cintigrafía de perfusión miocárdica}

Talio-201: El TI es un catión similar al potasio por lo cual entra y sale del miocito usando la bomba $\mathrm{Na}-\mathrm{K}$ ATPasa. Su emisión es de baja energía y por su vida media de 72 horas no se utilizan dosis mayores de $185 \mathrm{MBq}(5 \mathrm{mCi})$. Redistribuye si se inyecta en estrés. Los territorios con isquemia tienen un lavado más lento, lo que permite su pesquisa. Si se inyecta en reposo, los territorios hipoperfundidos pero viables también aumentan de concentración. Hay protocolos de viabilidad con reinyección del fármaco en reposo. Tecneciados (Sestamibi-Tc-99m): El Tc-99m tiene vida media de 6 horas y emisión gamma de $140 \mathrm{KeV}$. Se obtienen con estos radiofármacos excelentes imágenes tomográficas pues se pueden usar dosis mayores; además, la emisión gamma del Tc-99m tiene buenas características energéticas. Entran en la célula miocárdica dependiendo del flujo y de la indemnidad de la membrana y quedan atrapados a nivel del citoplasma. Requieren dos inyecciones: en estrés y reposo. Estas imágenes se pueden gatillar con la onda R del ECG, para evitar el movimiento y la visualización algo difusa de los bordes miocárdicos. FDG: La fluor deoxiglucosa marcada con $\mathrm{F}-18$ es un análogo de la glucosa, que es captado y fosforilado, pero que no entra al ciclo oxidativo y permanece atrapada como glucosa $6 \mathrm{P}$ intracelular. El fluor-18 es producto de ciclotrón, tiene vida media corta de aproximadamente dos horas y la energía liberada en la desintegración del positrón que emite es alta (dos gammas opuestos en 180 grados de sobre $500 \mathrm{KeV}$ ).

Tipos de estrés utilizados en cardiología nuclear Protocolos de ejercicio: Se utiliza el de Bruce o Bruce modificado en banda continua, en forma similar a la prueba de esfuerzo tradicional. Al alcanzar los parámetros hemodinámicos máximos previstos, según edad, se inyecta el radioisótopo. Esta prueba la efectúa un cardiólogo experimentado. La captación relativa de ejercicio que representa en parte el flujo de los territorios miocárdicos se compara con la captación del paciente en reposo.

Vasodilatadores coronarios: Los más usados son el Dipiridamol y la Adenosina endovenosa. Ellos aumentan en forma indirecta y directa la adenosina endógena vasodilatando los lechos coronarios. Los pacientes con estenosis no logran la máxima dilatación respecto a los normales. Las diferencias relativas de flujo se aprecian como diferencias de captación isotópica que se comparan con las de reposo. Son muy seguros. Producen también vasodilatación de otros territorios lo que implica efectos no deseados como cefalea, mareo y bochornos. Su antídoto es la aminofilina. No debieran emplearse en pacientes con obstrucción bronquial dependiente de broncodilatadores.

Inótropos: La dobutamina es el fármaco más utilizado que obtiene un real aumento de la contracción, del doble producto (frecuencia cardiaca x presión arterial) y del flujo miocárdico. Se usa sólo si existe contraindicación de los vasodilatadores.

Una alternativa para el diagnóstico y evaluación de EC y pesquisa de viabilidad es la ecocardiografía de estrés, el protocolo preferido, actualmente, es en dosis relativamente altas para provocar isquemia miocárdica o con dosis bajas con acción inótropa positiva para viabilidad.

\section{Interpretación de los estudios de perfusión}

1. Captación homogénea en las paredes del VI sin cambio entre las fases de estrés y reposo: Normal.

2. Defectos fijos en ambas fases: Infarto de miocardio.

3. Defecto reversible, es decir, defecto en estrés que se normaliza o disminuye significativamente en reposo: Isquemia.

4. Defectos parcialmente reversibles: Infarto de miocardio con isquemia residual.

5. Defecto en reposo que en imagen de redistribución 

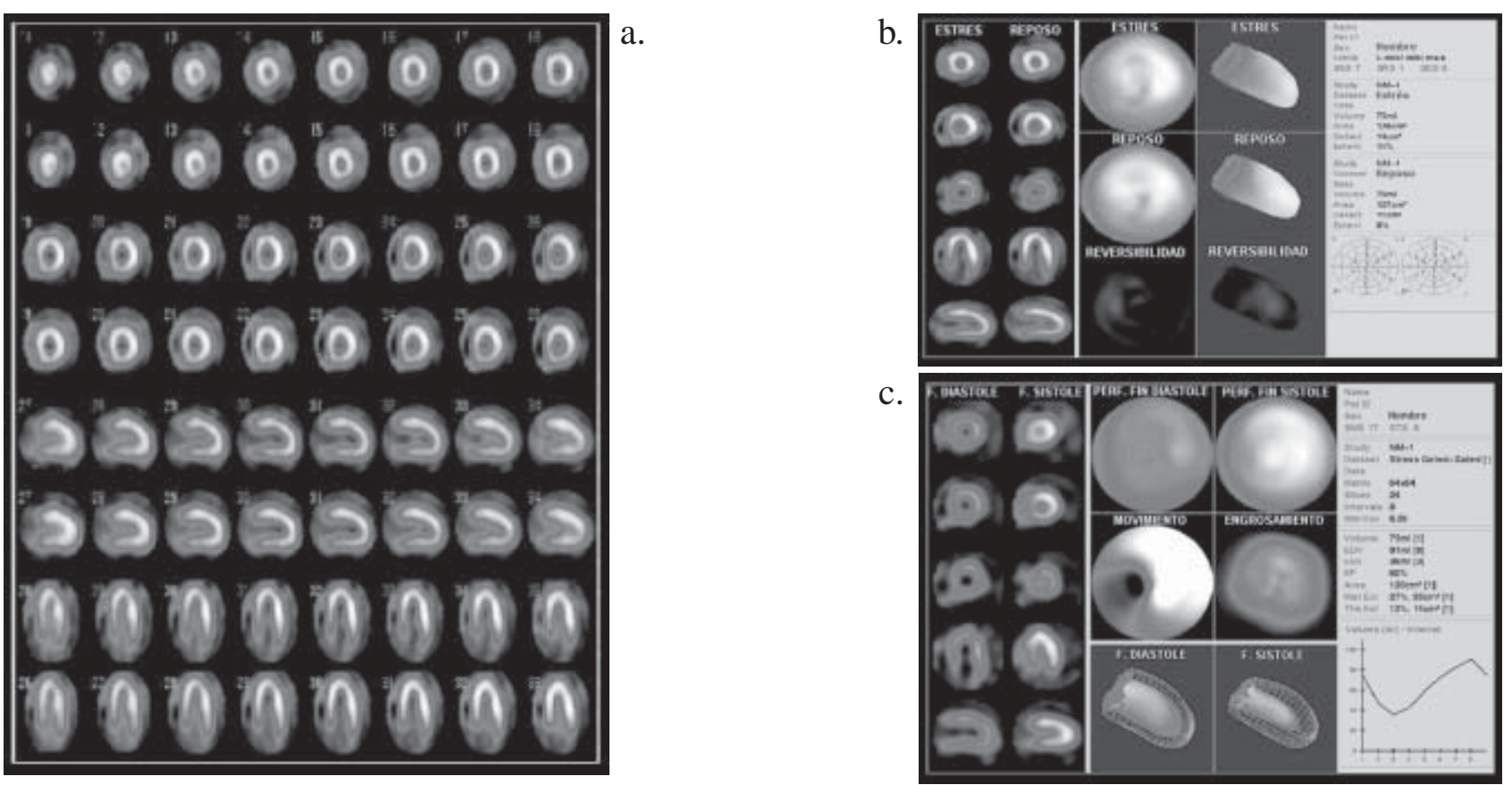

Figura 1 a,b,c. SPECT de perfusión miocárdica con Sestamibi-Tc99m en un hombre mayor con sospecha de EC. Estudio sin evidencias de isquemia. a.- Fases de estrés y reposo entre las cuales no se observan cambios significativos. Las columnas horizontales primera, tercera, quinta y séptima corresponden a la fase de estrés, y las segunda, cuarta, sexta y octava a la fase de reposo. Las proyecciones en eje corto (axial) están representadas en las columnas primera a cuarta y las de eje largo en las columnas quinta a octava. b.- Imágenes tridimensionales del ventrículo izquierdo y su perfusión en ambas fases. A derecha se representan los datos de cuantificación del volumen de la cavidad ventricular izquierda y el área de las paredes del miocardio de este ventrículo en estrés y reposo. c.- Estudio gatillado que muestra imágenes de la cavidad y volúmenes en sístole y diástole con motilidad y fracción de eyección normal. En el ángulo inferior derecho se representa la curva de volumen.
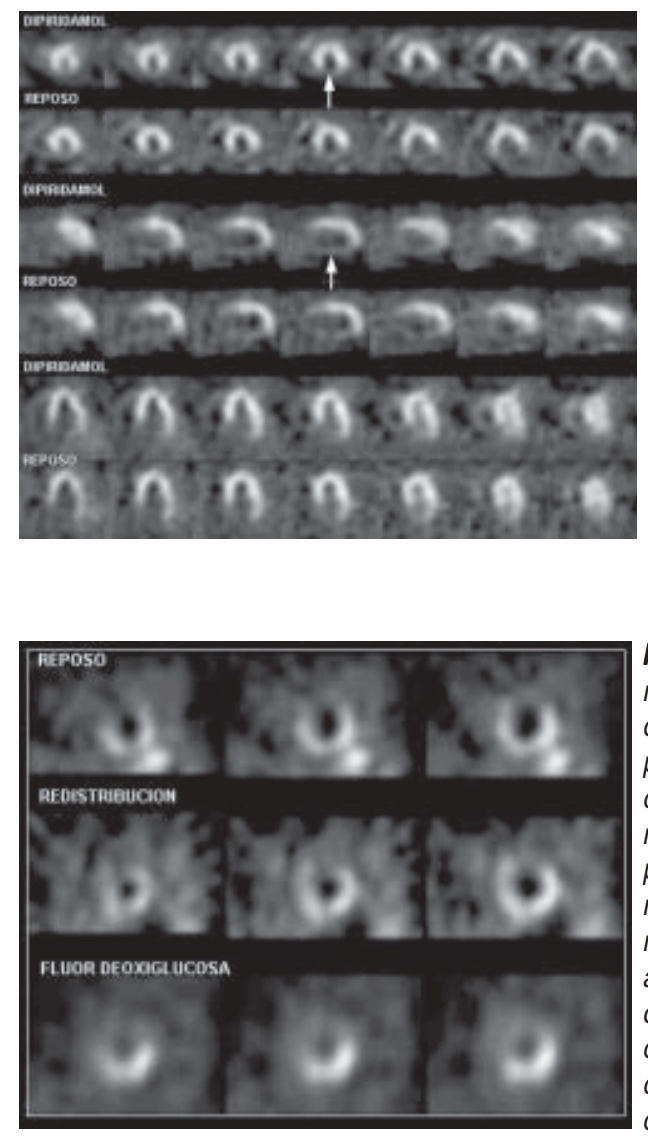

Figura 2. Estudio con Talio201 bajo acción de Dipiridamol (columnas primera tercera y quinta) y en reposo (columnas segunda, cuarta y sexta). Evaluación de riesgo pre-operatorio de gran cirugía no cardiaca en paciente coronario con antecedente de cirugía coronaria de puentes. No se demostró evidencias de isquemia, pero se pesquisó un infarto de cara inferior (flechas).

Figura 4. Infarto reciente de miocardio estudiado con protocolo de viabilidad (sin estrés). En la primera fila se observan los ejes cortos con TI-201 inyectado en reposo, en la segunda las imágenes posteriores del TI-201 en redistribución. Hay hipoperfusión marcada y extensa en la pared anterior correspondiente a una oclusión proximal de la arteria descendente anterior. La tercera fila corresponde a inyección de Flúordeoxiglucosa con defecto similar.

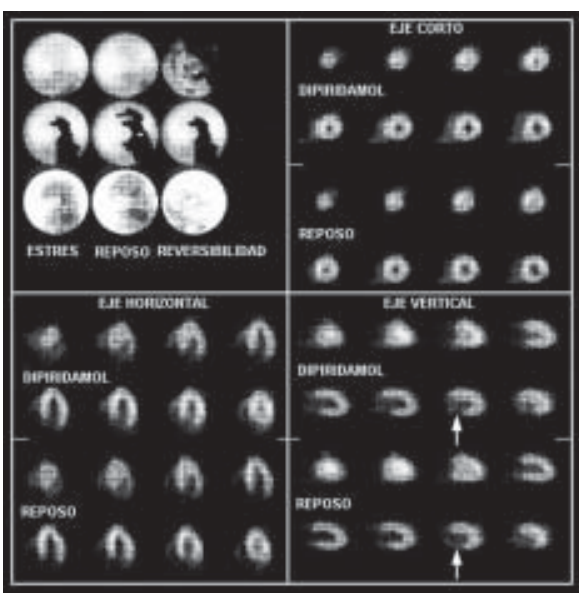

Figura 3. Estudio con TI-201 en paciente joven con dolor precordial atípico y antecedente de consumo de cocaína. Se hospitaliza pesquisándose hipokinesia basal. Se evalúa presencia de isquemia post infarto. Se observa severo defecto fijo inferior sin cambios significativos con Dipiridamol (flechas). La arteria coronaria derecha estaba ocluida. 
mejora su perfusión en protocolos de TI-201 o con captación mayor al $50 \%$ de la zona normal: Viable.

Como ya se mencionó el patrón estándar utilizado para estos estudios es la coronariografía.

\section{Conclusión}

Como puede apreciarse por la revisión anterior, el papel de la Medicina Nuclear es relevante en cardiología, tanto en la práctica clínica habitual, en especial, diagnóstico y manejo de la enfermedad coronaria, con emisores gamma (Talio-201 y Tecnecio-99m), y en la detección de viabilidad miocárdica también en miocardiopatía isquémica, tanto con compuestos emisores gamma como con positrones (Fluor-18). Por otro lado, en la investigación básica de fisiopatología cardiaca también se utilizan diversos emisores de positrones, tema muy interesante, que requiere mayor desarrollo.

\section{Bibliografia}

1. ACC/AHA Guidelines for the Management of Patients With Acute Myocardial Infarction. A Report of the American College of Cardiology/American Heart Association Task Force on Practice Guidelines. (Committee on Management of Acute Myocardial Infarction). J Am Coll Cardiol 1996; 28:1328-1428.

2. ACC/AHA Task Force Report Guidelines for clinical use of cardiac radionuclide imaging. Report of the American College of Cardiology /American Heart association Task Force on assessment of diagnostic and therapeutic cardiovascular procedures (Committee on radionuclide imaging) developed in collaboration with the American Society of Nuclear Cardiology. J Am Coll Cardiol 1995; 25:521-547.

3. Bax J, Visser F, Van Lingen A, y col. Feasibility of assessing regional myocardial uptake of $\mathrm{F}-18$ fluorodeoxyglucose using Single Photon Emission Computed Tomography. Eur Heart J. 1993; 14: 16751682.

4. Bax JJ, Wijns W, Cornel JH y col. Accuracy of currently available techniques for prediction of functional recovery after revascularization in patients with left ventricular dysfunction due to chronic coronary artery disease: comparison of pooled date. J Am Coll Cardiol 1997; 30:1451-1460.

5. Beller George, Risk stratification after acute myocardial infarction by using noninvasive radionuclide imaging, in Nuclear Cardiology, Zaret B. and Beller G. Editors, Saint Louis Missouri, Mosby 1999; Pag.451-467.

6. Burt RW, Perkins OW, Oppenheim BE., et al. Comparison of F18-FDG SPECT, F18-FDG PET and rest thallium 201 SPECT for detection of myocardial viability. J Nucl Med 1995; 36: 176-179.

7. Candell-Riera J, Castell-Conesa J González JM and Roselló-Urgel J. Eficacia del esfuerzo-reposo con $99 \mathrm{mTc}-\mathrm{MIBI}$ en la predicción de la recuperación de la función contráctil posrevascularización. Resultados del protocolo multicéntrico Español. Rev Esp Cardiol 2000; 53:903-910.
8. Coolly J, Hake AH, Iskandrian AS. Homodynamic, angiographic and scintigraphic correlation of positive exercise electrocardiograms: emphasis on strongly positive exercise electrocardiograms. J Am Coll Cardiol 1983; 2: 21-27.

9. Dilsizian V, Perrone-Filardi P, Arrighi JA, y col.. Concordance and discordance between stress-redistribution-reinjection and rest-redistribution thallium imaging for assessing viable myocardium. Comparison with metabolic activity by positron emission tomography. Circulation 1993 ; 88: 283-293.

10. Gibbons RJ, Chatterjee K, Daley J, Douglas JS, y col. ACC/AHA/ACP-ASIM guidelines for the management of patients with chronic stable angina: a report of the American College of Cardiology/American Heart Association Task Force on Practice Guidelines. J Am Coll Cardiol 1999;33:2092-2197.

11. González P, Massardo T, Muñoz A y col. Is the addition of ECG gating to technetium-99m Sestamibi of value in the assessment of myocardial viability? Eur J Nucl Med 1996; 23:1315-1322.

12. González P., Pawlak M., Raggio R y col. Utilidad de la prueba de esfuerzo con Talio 201 en cardiopatía coronaria. Rev Med Chile 1982; 110: 839-847.

13. Inglese $E$, Brambilla $M$, Dondi $M$, et al. Assessment of myocardial viability after Thallium 201 reinjection or restredistribution imaging: A multicenter study. J Nucl Med 1995; 36: 555-563.

14. Iskandrian A, Hakki A, Kane SA., et al. Rest and redistribution thallium myocardial scintigraphy to predict improvement in left ventricular function after coronary arterial bypass grafting. Am J Cardiol 1983; 51: 13121316.

15. Iskandrian AS, Heo J, Schelbert HR. Myocardial viability: methods of assessment and clinical relevance. Am Heart J 1996 Dec;132(6):1226-1235.

16. L'Abbate A., Biagini A., Michelassi C, et al. Myocardial kinetics of thallium and potassium in man. Circulation 1979, 60:776-785.

17. Lee KS, Mark TH, Cook SA, et al. Prognosis of patients with left ventricular dysfunction, with and without viable myocardium after myocardial infarction. Circulation 1994; 90: 2687-2694.

18. Pierard L, De Landsheere C, Berthe C, et al. Identification of viable myocardium by echocardiography during dobutamine infusion in patients with myocardial infarction after thrombolytic therapy: Comparison with positron emission tomography. JACC 1990:1021-1031.

19. Port SC. Imaging Guidelines for Nuclear Cardiology Procedures. Part 2. J Nucl Cardiol 1999; 6: G47-84.

20. Schwaiger M, Brunken R, Grover-McKay M, et al. Regional myocardial metabolism in patients with acute myocardial infarction assessed by PET. J Am Coll Cardiol 1986; 8: 800-808.

21. Strauss HW, Harrison K, Langan JK, et al. Thallium 201 from myocardial imaging. Relation of Thallium 201 to regional myocardial perfusion. Circulation 1975; 51:641-645.

22. Tamaki N, Ohtani H, Yonekura $\mathrm{Y}$ et al. Viable myocardium identified by reinjection thallium-201 imaging: comparison with regional wall motion and metabolic activity on FDG-PET. J Cardiol 1992; 22:283-293. 